\title{
Exogenous C-type natriuretic peptide infusion ameliorates unilateral ureteral obstruction-induced tubulointerstitial fibrosis in rats
}

\author{
Peng $\mathrm{Hu}^{1}$, Xiao Cen Zhang ${ }^{1,2}$, Hai Bo Kong ${ }^{1}$, Xun Xia ${ }^{1}$, Bo Hu${ }^{1}$ and Yuan Han Qin $^{3}$
}

\begin{abstract}
Although many experimental therapeutic roles for C-type natriuretic peptide (CNP) have been documented in the field of cardiovascular and pulmonary-vascular disease, the therapeutic uses of CNP to nephropathies are not as well documented. In this study, we established a rat model of unilateral ureteral obstruction (UUO) to observe the beneficial effects of CNP on tubulointerstitial fibrosis (TIF). In UUO rats, CNP administration induced a significant increase in plasma CNP levels, and caused a significant decrease in blood urea nitrogen and creatinine levels. In addition, CNP infusion also alleviated the pathological lesions and collagen IV accumulation in the obstructed kidneys through downregulation of tissue inhibitor of metalloproteinase-1 (TIMP-1) and TIMP-2 expression. In conclusion, exogenous CNP infusion can ameliorate UUO-induced TIF in rats. However, the use of CNP as a therapeutic agent requires further evaluation before being considered for human TIF.
\end{abstract}

Laboratory Investigation (2015) 95, 263-272; doi:10.1038/labinvest.2014.149; published online 1 December 2014

C-type natriuretic peptide (CNP) shares sequence homology with the endocrine cardiac peptides, atrial natriuretic peptide (ANP), and brain natriuretic peptide (BNP). They comprise a ring structure of 17 amino acids linked by a cysteine disulfide bridge that is essential for receptor binding and bioactivity. However, unlike the other members of natriuretic peptide family, CNP selectively binds to the transmembrane natriuretic peptide receptor (NPR)-B and subsequently leads to a large increase in intracellular cyclic guanosine monophosphate (cGMP). ${ }^{1} \mathrm{CNP}$ was first isolated from pigs, ${ }^{2}$ but CNP immunoreactivity has been found in a wide variety of tissues, such as vascular endothelium, heart, bone, adrenal, and reproductive glands. ${ }^{3-7}$ CNP is also believed to be produced locally in the kidney. Several studies have documented a significant arteriovenous gradient for CNP across both the human and animal kidneys, indirectly indicating renal extraction of this peptide. ${ }^{8,9}$ Our previous immunohistochemical study showed that CNP was predominantly present in tubular epithelial cells, including proximal, distal, and medullary collecting duct cells; moreover, CNP immunoreactivity was also observed in glomeruli. ${ }^{10}$ In pathological circumstances, either urinary levels or renal tissue expression of CNP may act as a sensitive marker of nephropathies. In a recent study, we established a unilateral ureteral obstruction (UUO) model in Wistar rats, and observed that expression of CNP mRNA and protein in the obstructed kidneys tended to be higher immediately after ligation, and then declined progressively. ${ }^{11}$ An expansion of extracellular fluid volume may account for the early elevation in CNP expression. ${ }^{12}$ However, epithelial-mesenchymal transition, combined with the paradoxical expressions of NPR-C and neutral endopeptidase (NEP), may account for the decline of CNP in the advanced stages of nephropathies. ${ }^{10,11}$

In contrast to regulation of blood pressure and body fluid homeostasis, the known physiological actions of CNP include local vasorelaxation, inhibition of organ remodeling, acceleration of reendothelialization, and antagonism of the reninangiotensin-aldosterone system. ${ }^{13-16}$ Izumiya et al ${ }^{17}$ examined the effects of exogenous CNP administration $(0.05 \mu \mathrm{g} / \mathrm{kg} / \mathrm{min}$ $\times 2$ weeks) on C57BL/6 mice with angiotensin II pretreatment, and found that angiotensin II-induced upregulation of collagen-I (Col-I), Col-III, and glyceraldehyde-3-phosphate dehydrogenase oxidase 4 in hearts was significantly suppressed by exogenous CNP infusion. In another study

\footnotetext{
${ }^{1}$ Department of Pediatrics, The First Affiliated Hospital of Anhui Medical University, Hefei, People's Republic of China; ${ }^{2}$ Department of Pediatrics, People's Hospital of Fuyang City, Fuyang, People's Republic of China and ${ }^{3}$ Department of Pediatrics, The First Affiliated Hospital of Guangxi Medical University, Nanning, People's Republic of China

Correspondence: Dr P Hu, PhD, MD, Department of Pediatrics, The First Affiliated Hospital of Anhui Medical University, No. 218, Ji-Xi Road, Hefei 230022, People's Republic of China.

E-mail: hupeng28@aliyun.com

Received 22 March 2014; revised 28 July 2014; accepted 12 September 2014
} 
conducted by Itoh et al, ${ }^{18}$ continuous infusion of CNP $(0.75 \mu \mathrm{g} / \mathrm{h} \times 2$ weeks $)$ had beneficial effects on hemodynamics and survival in Wistar rats suffering from monocrotaline-induced pulmonary hypertension, via regeneration of pulmonary endothelium, inhibition of endothelial cell apoptosis, and prevention of monocyte/macrophage infiltration and fibrinolysis impairment. Although many of the experimental therapeutic roles for CNP have been documented in cardiovascular and pulmonary-vascular disease, the therapeutic uses of CNP in nephropathies are not as well studied.

\section{MATERIALS AND METHODS Animals and Treatment}

Male Wistar rats weighting 190 to $250 \mathrm{~g}$ were housed at an ambient temperature of $23 \pm 1{ }^{\circ} \mathrm{C}$ and exposed to a daily $12-\mathrm{h}$ light/dark cycle (light on at 0700 to 19:00 h) with free access to tap water and a pellet diet. Animals were treated humanely using approved procedures in accordance with the guidelines of the Institutional Animal Use and Care Committee of Anhui Medical University. The fasted animals were operated under intraperitoneal pentobarbital anesthesia $(60 \mathrm{mg} / \mathrm{kg}$ body weight) and sterile conditions. UUO rats underwent left proximal ureteral ligation with 4-0 silk at the junction of the upper with the two lower thirds of its length. The ureter was cut between the ligatures to prevent retrograde urinary tract infection. They were divided randomly into the UUO group and the UUO treated with CNP group. Thirty rats were used for each group. To the UUO rats treated with CNP, UUO operation was followed by the first dose of CNP infusion, and caudal vein was the infusion given. A continuous infusion of CNP $\quad(0.05 \mu \mathrm{g} / \mathrm{kg} / \mathrm{min} \times 1 \mathrm{~h}, \quad 1 \mu \mathrm{g} / \mathrm{kg} / \mathrm{min}=0.5 \mathrm{nM} / \mathrm{kg} / \mathrm{min}$, Sangon Biotech, Shanghai, China) was given once a day from the day of the disease induction until the day of killing. The other 30 animals were the sham-operation group, and underwent a sham laparotomy with ureteric manipulation through a midline incision. Subsequently, animals in each group were separated into five experimental subgroups, anesthetized by intraperitoneal pentobarbital injection, and killed by heart puncture at $72 \mathrm{~h}, 1$ week, 2 weeks, 3 weeks, and 1 month after ligation, respectively. The obstructed kidneys of UUO rats and the left kidneys of sham-operation group were harvested.

\section{Radioimmunoassay}

Blood samples were taken from abdominal aorta. CNP immunoreactivity was determined by a radioimmunoassay (crossreactivity to human ANP, BNP, and DNP $<1 \%$; Phoenix pharmaceuticals, USA) after extraction as previously described. ${ }^{19}$ Blood samples were collected in disodium EDTA vacutainers containing aprotinin $(500 \mathrm{KIU} / \mathrm{ml}$ of blood), centrifuged at $1600 \mathrm{~g}$ for $15 \mathrm{~min}$ at $4{ }^{\circ} \mathrm{C}$, and stored at $-80^{\circ} \mathrm{C}$ until assayed for CNP. Then, $2 \mathrm{ml}$ of the plasma was passed through Sep-Pack C18 cartridges and eluted with $5 \mathrm{ml}$ $60 \%$ acetonitrile containing $0.1 \%$ trifluoracetic acid. The eluate was lyophilized and reconstituted for radioimmunoassay. Duplicate samples were tested for radioimmunoassay. Serial dilutions (1:1, 1:2, 1:4, 1:8, and 1:16) of plasma samples were subjected to CNP immunoreactivity. The correlation coefficients between the levels of CNP immunoreactivity and the degree of dilution were $r=0.98$ and $r=0.99$. The intraand interassay coefficients of variation were 5.0 and $10.0 \%$ for plasma CNP immunoreactivity. The sensitivity and specificity were found to be $97.2 \%$ and $96.5 \%$, respectively.

\section{Laboratory Analysis}

All rats were placed in metabolic cages and urine was collected for $24 \mathrm{~h}$. Urinary protein concentration (UP) from $24 \mathrm{~h}$ urine sample was determined by biuret colorimetric method (Boehringer Mannheim, Italy). Serum total protein (TP), albumin (Alb), blood urea nitrogen (BUN), and creatinine (Cr) were measured by standard enzymatic method (Randox, UK).

\section{Renal Morphology}

At harvest, each kidney was washed with saline, blotted dry on gauze, and weighed. Midcoronal kidney sections were fixed in $4 \%$ paraformaldehyde and embedded in paraffin. Paraffin sections ( $4 \mu \mathrm{m}$ thick) were stained with hematoxylin and eosin, and examined independently by two pathologists blinded to the experimental design. Stereological evaluation was determined in each section selected at random, and 10 rectangular fields were examined under $\times 400$ magnification. Glomerular area (GA) was as follows: $\mathrm{GA}=\pi r_{1}{ }^{2}+\pi r_{2}{ }^{2}+$ $\pi r_{3}^{2}+\ldots+\pi r_{n}^{2}$. The radius of each glomerulus was determined by halving the diameter, and this would be easier to measure in the profile rather than trying to estimate its center point. Tubulointerstitial area (TA) can be estimated by subtracting total GA from rectangular field (RA), TA= RA - GA (Figure 1a). Renal glomerulus is a spherical capillary network. When an entire glomerulus is cut into different sections, the maximum radius $\left(r_{\max }\right)$ is very close to the actual radius of glomerulus. Glomerular volume (GV) was as follows: $\mathrm{GV}=4 \pi r_{\max }{ }^{3} / 3$ (Figure $1 \mathrm{~b}$ ). ${ }^{20}$

\section{Real-Time PCR}

Total RNA was extracted from renal tissue using TRIzol reagent (Invitrogen, USA). Ultraviolet spectrophotometer measuring absorbance and agarose gel electrophoresis confirmed that there had been no degradation of RNA. Specific primers were synthesized according to the published sequences. The primers of CNP, MMP-2, MMP-9, TIMP-1, TIMP-2, Col-IV, and glyceraldehyde-3-phosphate dehydrogenase (GAPDH) are shown in Table $1 .{ }^{21-26}$ From the renal tissue of each rat, $1 \mu \mathrm{g}$ total RNA was reverse transcribed into cDNA with an ExScript RT reagent kit (Takara Biotechnology, Dalian, China). CNP, MMP-2, MMP9, TIMP-1, TIMP-2, and Col-IV were amplified with SYBR Premix Ex Taq (Takara Biotechnology). Gene expression of GAPDH was also measured in each sample and used as an internal control for loading and reverse transcription 
a

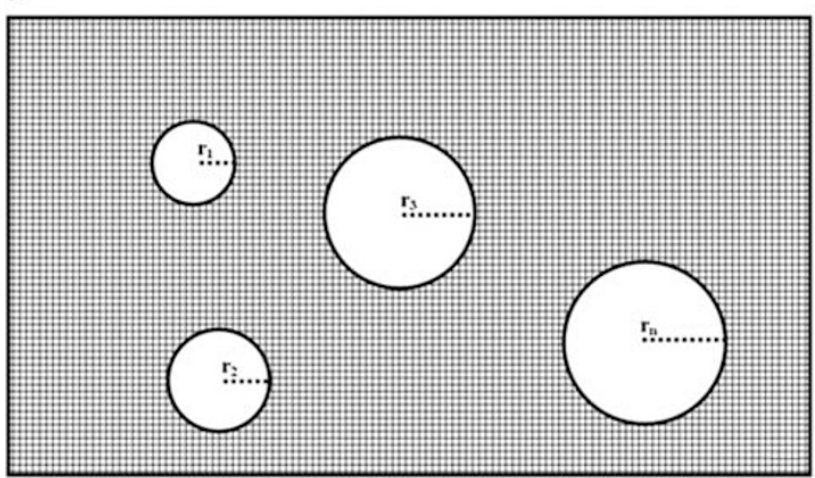

b

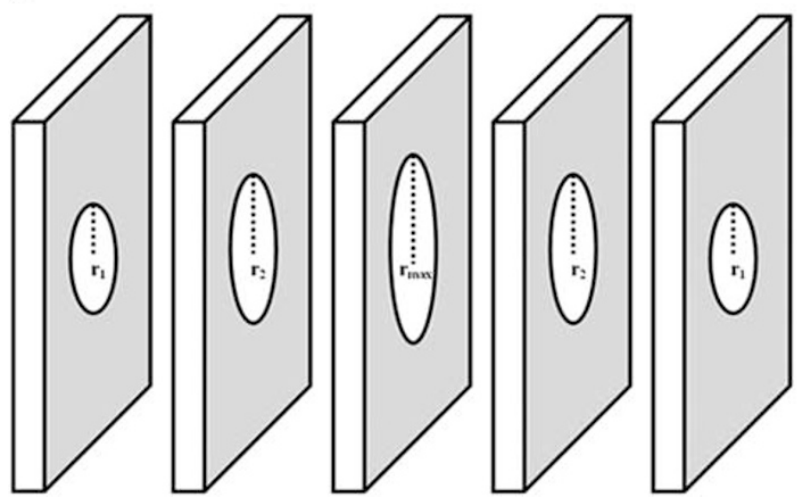

Figure 1 Stereological evaluation of TIP and GV.

$\mathrm{TA}=\mathrm{RA}-\left(\pi r_{1}{ }^{2}+\pi r_{2}{ }^{2}+\pi r_{3}^{2}+\ldots+\pi r_{n}{ }^{2}\right)(\mathbf{a}) ; \mathrm{GV}=4 \pi r_{\max }{ }^{3} / 3(\mathbf{b})$.

efficiency. Each sample analysis was repeated three times. The average threshold cycle (Ct, the cycles of template amplification to the threshold) was worked out as the value of each sample. Relative quantitative $2^{-\Delta \Delta \mathrm{Ct}}$ was used to compare the mRNA expression. ${ }^{27}$

\section{Protein Extraction and Western Blot Analysis}

Renal tissues were homogenized in ice-cold buffer $(40 \mathrm{mM}$ $\mathrm{KCl}, 10 \mathrm{mM}$ Hepes, $\mathrm{pH} 7.9,3 \mathrm{mM} \mathrm{MgCl}_{2}, 5 \%$ glycerol, $0.5 \mu \mathrm{g} / \mathrm{ml}$ leupeptin, $0.1 \mu \mathrm{g} / \mathrm{ml}$ aprotinin, $1.5 \mu \mathrm{g} / \mathrm{ml}$ pepstatin, and $100 \mu \mathrm{g} / \mathrm{ml}$ phenylmethylsulphonyl fluoride) with a Polytron homogenizer for $15-20 \mathrm{~s}$. The homogenates were centrifuged at $500 \times \mathrm{g}$ for $10 \mathrm{~min}$ at $4{ }^{\circ} \mathrm{C}$ and the supernatants were recentrifuged at $12000 \mathrm{~g}$ for $60 \mathrm{~min}$ at $4{ }^{\circ} \mathrm{C}$. The pellets were resuspended in $0.5 \mathrm{ml}$ homogenizing buffer containing $0.5 \%$ Nonidet P-40 (Boster, Wuhan, China) and total protein concentration of the supernatant was determined by the dyebinding method using bovine serum albumin as the standard. Aliquots of the solubilized membranes $(40 \mu \mathrm{g})$ were dried in speed-vac and resuspended in $20 \mu \mathrm{l}$ sodium dodecyl sulfate (SDS) loading buffer. The protein samples were heated to $70{ }^{\circ} \mathrm{C}$ for $15 \mathrm{~min}$ and loaded on SDS polyacrylamide gels (Bio-Rad, Beijing, China) and SDS-PAGE separated at $70 \mathrm{~V}$ over a $90-\mathrm{min}$ period. The protein bands were electrophoretically transferred to nitrocellulose membranes (Bio-
Table 1 List and sequence of primers

\begin{tabular}{|c|c|c|}
\hline Variable & Sequence & Reference number \\
\hline CNP & & 21 \\
\hline Sense & 5'-AACATCCCAGACCGCTCATG-3' & \\
\hline Antisense & 5'-CAAGAAGGGCTTGTCCAAAGG-3' & \\
\hline$M M P-2$ & & 22 \\
\hline Sense & 5'-ACTCCAGTTAAAGGCAGCATCTAC-3' & \\
\hline Antisense & 5'-CAGGGAATGAGTACTGGGTCTATT-3' & \\
\hline MMP-9 & & 23 \\
\hline Sense & 5'-CGCAAGCCTCTAGAGACCAC-3' & \\
\hline Antisense & 5'-TGGGGGATCCGTGTTATTA-3' & \\
\hline TIMP-1 & & 24 \\
\hline Sense & 5'-GGCAGTGATGTGCAAATTTCC-3' & \\
\hline Antisense & 5'-CGCAGCGAGGAGTTTCTCAT-3' & \\
\hline TIMP-2 & & 25 \\
\hline Sense & 5'-GGTGCCCATTGATGCTCTTC-3' & \\
\hline Antisense & 5'-CGCTTAGCATCACCCAGAAGA-3' & \\
\hline Col-IV & & 26 \\
\hline Sense & 5'-GAGGGTGCTGGACAAGC-3' & \\
\hline Antisense & 5'-TAAATGGACTGGCTCGGAATTC-3' & \\
\hline GAPDH & & 23 \\
\hline Sense & 5'-TGTGAGGGAGATGCTCAGTG-3' & \\
\hline Antisense & 5'-GGCATTGCTCTCAATGACAA-3' & \\
\hline
\end{tabular}

Rad, Beijing, China) and the nonspecific binding sites were blocked with buffer A (20 mM Tris-HCl, pH 7.5, $0.5 \mathrm{mM}$ $\mathrm{NaCl}$, and $0.1 \%(\mathrm{v} / \mathrm{v})$ Tween-20) containing $10 \%$ skimmed milk for $12 \mathrm{~h}$ at $4{ }^{\circ} \mathrm{C}$. The membranes were incubated for $60 \mathrm{~min}$ at room temperature in buffer A containing 5\% skimmed milk with anti-CNP, MMP-2, MMP-9, TIMP-1, TIMP-2, and Col-IV rabbit antibodies (1:500 dilution). Membranes were washed three times $(10 \mathrm{~min}$ each) with buffer A, followed by incubation with horseradish peroxidase-conjugated anti-rabbit IgG antibodies (1:1000 dilution; Boster, Wuhan, China) in buffer A containing 5\% skimmed milk for $60 \mathrm{~min}$, followed by a further three washes with buffer A as described previously. The immunoreactive bands were visualized using the enhanced chemiluminescence kit (Amersham, Buckinghamshire, UK) and exposed on radiographic films for variable periods.

\section{Statistical Analyses}

All values are expressed as mean \pm s.e.m. Comparison of mean values between groups was made using one-way 
ANOVA, and post hoc analysis was calculated using the Student-Newman--Keuls test. A value of $P<0.05$ was considered significant. Statistical analysis was performed using the statistical package for social studies SPSS version 11.5.

\section{RESULTS}

The baseline levels of plasma CNP in the sham-operation group were $1.30 \pm 0.23,1.15 \pm 0.16,1.19 \pm 0.13,1.28 \pm 0.27$, and $1.08 \pm 0.14 \mathrm{pmol} / \mathrm{l}$ at $72 \mathrm{~h}, 1$ week, 2 weeks, 3 weeks, and 1 month after ligation, respectively. UUO did not affect the baseline levels of plasma CNP at each time point $(P>0.05)$. The plasma CNP levels in the UUO group were $1.23 \pm 0.19$, $0.98 \pm 0.11,1.06 \pm 0.16,1.21 \pm 0.21$, and $0.93 \pm 0.10 \mathrm{pmol} / \mathrm{l}$ at $72 \mathrm{~h}, 1$ week, 2 weeks, 3 weeks, and 1 month after ligation, respectively. Nevertheless, to the UUO rats, administration of CNP induced a significant increase in the plasma CNP levels at each time point $(P<0.05)$. The plasma CNP levels in the UUO treated with CNP group were $13.62 \pm 3.45,11.78 \pm 3.12,9.54 \pm$ $2.83,12.15 \pm 3.51$, and $8.91 \pm 2.26 \mathrm{pmol} / \mathrm{l}$ at $72 \mathrm{~h}, 1$ week, 2 weeks, 3 weeks, and 1 month after ligation, respectively.

Biochemical parameters among the three groups are presented in Table 2. At the time of killing, the UP levels were raised gradually, but significantly, in the UUO rats from
$72 \mathrm{~h}$ to 1 month after ligation in comparison with their sham-operation counterparts $(P<0.05)$. No significant differences in the plasma TP levels were observed between the sham-operation group and the UUO group at all time points $(P>0.05)$, but Alb was remarkably decreased in the UUO rats at 3 weeks and 1 month after ligation $(P<0.05)$. UUO rats exhibited significant higher BUN than those in the shamoperation group from 2 weeks to 1 month after ligation $(P<0.05)$, and significant higher $\mathrm{Cr}$ than those in the sham-operation group from 1 week to 1 month after ligation $(P<0.05)$. Although exogenous CNP infusion could significantly decrease the BUN and Cr levels in the UUO rats from 2 weeks to 1 month after ligation $(P<0.05)$, the other biochemical disorders in the UUO treated with CNP group were still observed, compared with the corresponding sham-operation group $(P<0.05)$.

Representative histology images of the kidneys obtained from the three groups are shown in Figure 2. Sections derived from the sham-operation group had normal appearance at all time points. However, all morphological lesions observed in the obstructed kidneys became more aggravated with time. After $72 \mathrm{~h}$ of ligation, renal damage was limited to a reduction of peritubular capillaries, tubular atrophy, and widen

Table 2 Biochemical parameters among the three groups

\begin{tabular}{|c|c|c|c|c|c|}
\hline & UP (mg/l) & $\mathrm{TP}(\mathrm{g} / \mathrm{l})$ & Alb $(g / l)$ & BUN (mmol/l) & $\mathrm{Cr}(\mu \mathrm{mol} / \mathrm{l})$ \\
\hline \multicolumn{6}{|c|}{ Sham-operation group } \\
\hline 1 Week & $477.98 \pm 95.60$ & $64.88 \pm 4.12$ & $33.45 \pm 4.85$ & $8.02 \pm 3.65$ & $24.67 \pm 4.80$ \\
\hline 2 Weeks & $497.10 \pm 86.36$ & $62.04 \pm 3.25$ & $30.60 \pm 4.60$ & $7.46 \pm 1.96$ & $24.20 \pm 5.71$ \\
\hline 1 Month & $458.59 \pm 53.82$ & $61.95 \pm 2.28$ & $32.48 \pm 2.57$ & $7.84 \pm 1.54$ & $24.00 \pm 4.62$ \\
\hline \multicolumn{6}{|l|}{ UUO group } \\
\hline $72 \mathrm{~h}$ & $567.30 \pm 74.70^{\mathrm{a}}$ & $64.71 \pm 2.07$ & $31.76 \pm 5.34$ & $8.95 \pm 2.47$ & $28.60 \pm 4.08$ \\
\hline 1 Week & $676.24 \pm 79.12^{a}$ & $63.55 \pm 4.33$ & $30.43 \pm 2.34$ & $10.20 \pm 2.43$ & $35.00 \pm 4.00^{\mathrm{a}}$ \\
\hline \multicolumn{6}{|c|}{ UUO treated with CNP group } \\
\hline $72 \mathrm{~h}$ & $587.13 \pm 84.65^{\mathrm{a}}$ & $58.41 \pm 3.98$ & $29.66 \pm 4.74$ & $7.91 \pm 3.30$ & $28.04 \pm 5.67$ \\
\hline 1 Week & $681.20 \pm 80.03^{\mathrm{a}}$ & $61.16 \pm 4.05$ & $29.19 \pm 4.57$ & $8.45 \pm 3.65$ & $33.28 \pm 5.42^{\mathrm{a}}$ \\
\hline 2 Weeks & $699.77 \pm 78.25^{\mathrm{a}}$ & $56.38 \pm 4.47$ & $28.24 \pm 3.96$ & $10.40 \pm 3.77^{\mathrm{a}, \mathrm{b}}$ & $37.50 \pm 5.45^{\mathrm{a}, \mathrm{b}}$ \\
\hline 3 Weeks & $843.96 \pm 92.81^{\mathrm{a}}$ & $58.19 \pm 4.73$ & $24.37 \pm 5.03^{\mathrm{a}}$ & $11.94 \pm 3.51^{a, b}$ & $41.17 \pm 6.80^{\mathrm{a}, \mathrm{b}}$ \\
\hline 1 Month & $860.74 \pm 87.22^{\mathrm{a}}$ & $56.42 \pm 5.02$ & $20.68 \pm 4.69^{a}$ & $14.66 \pm 4.15^{\mathrm{a}, \mathrm{b}}$ & $45.31 \pm 6.61^{\mathrm{a}, \mathrm{b}}$ \\
\hline
\end{tabular}

${ }^{a} P<0.05$, significantly different from the corresponding sham-operation group.

${ }^{\mathrm{b}} P<0.05$, significantly different from the corresponding UUO group. 

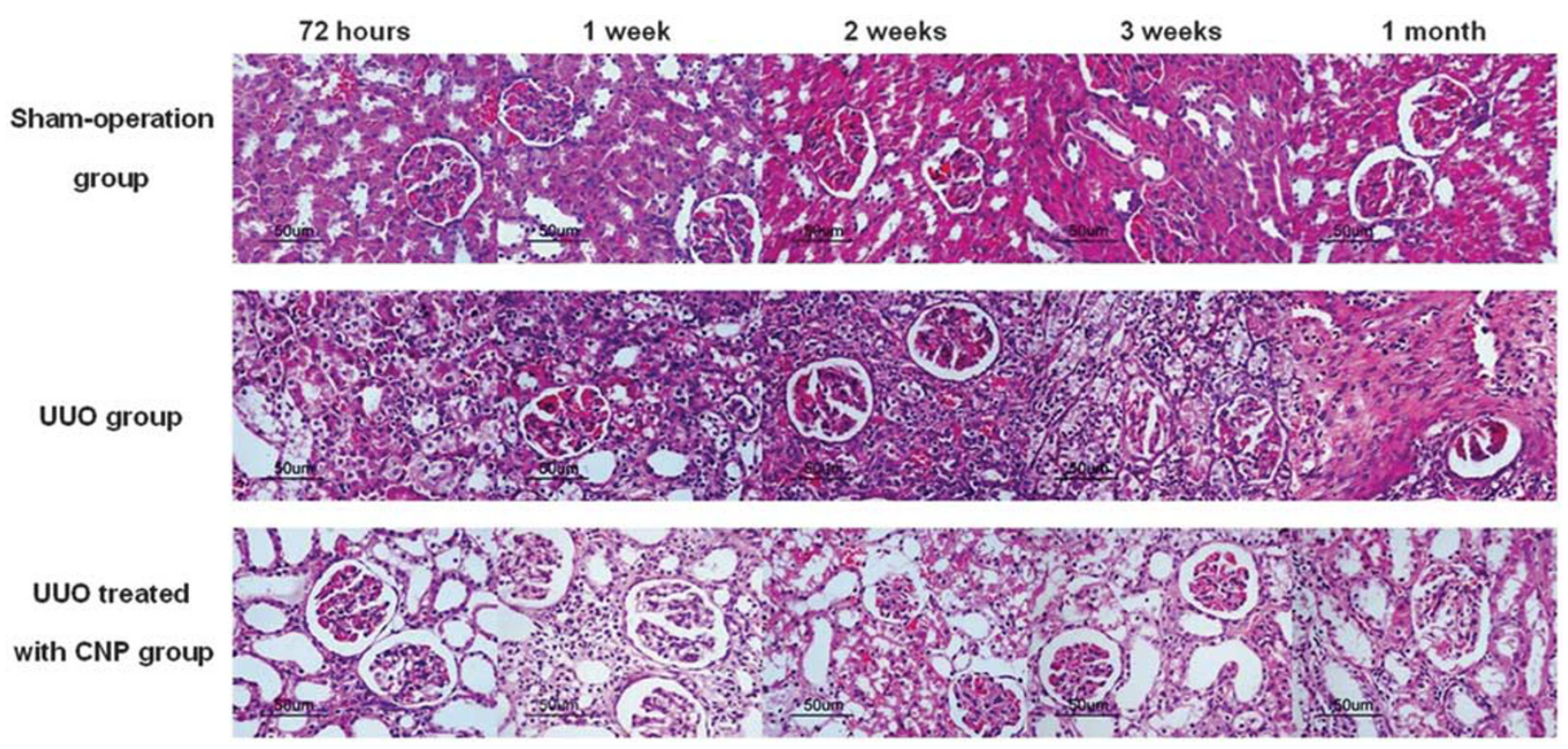

Figure 2 Representative histology images of the kidneys obtained from the three groups. Sections derived from the sham-operation group had normal appearance at all time points. However, all morphological lesions observed in the obstructed kidneys became more aggravated in time. After $72 \mathrm{~h}$ of ligation, renal damage was limited to a reduction of peritubular capillaries, tubular atrophy, and widen interstitial space, accompanied by an inflammatory cell infiltration in the interstitium. At 1,2, and 3 weeks after ligation, all lesions observed at earlier time point worsened and increased extracellular matrix (ECM) deposition became a prominent feature. Atrophic tubules were surrounded by a thickened, wrinkled basement membrane. Glomerular damage was limited to thickening of Bowman's capsule. At 1 month after ligation, ECM deposition increased further and the interstitial space of the obstructed kidneys was populated by numerous fibroblasts. The above pathological lesions were alleviated significantly in the UUO treated with CNP group.

interstitial space, accompanied by inflammatory cell infiltration in the interstitium. At 1, 2, and 3 weeks after ligation, all lesions observed at earlier time points worsened, and increased extracellular matrix (ECM) deposition became a prominent feature. Atrophic tubules were surrounded by a thickened, wrinkled basement membrane. Glomerular damage was limited to thickening of Bowman's capsule. At 1 month after ligation, ECM deposition was more obvious and the interstitial space of the obstructed kidneys was populated by numerous fibroblasts. The above pathological lesions were alleviated significantly in the UUO treated with CNP group, but tubular dilatation was prominent. At the time of killing, TA were raised gradually, but significantly, in the obstructed kidneys only at 1 month after ligation in comparison with the sham-operation group (31854.38vs $29642.35 \mu \mathrm{m}^{2}, P<0.05$ ). On the contrary, GA and GV were decreased progressively with time, but significantly, in the obstructed kidneys from 3 weeks to 1 month after ligation compared with the shamoperation group (3249.51 vs $4498.54 \mu \mathrm{m}^{2}$ at 3 weeks after ligation for GA; $2480.62 v s 4312.38 \mu \mathrm{m}^{2}$ at 1 month after ligation for GA; 116571.82 vs $148332.75 \mu \mathrm{m}^{3}$ at 3 weeks after ligation for GV; $111354.75 v s 150111.92 \mu \mathrm{m}^{3}$ at 1 month after ligation for $\mathrm{GV} ; P<0.05)$. Exogenous $\mathrm{CNP}$ infusion caused a significant decline of TA in the UUO treated with CNP group at 1 month after ligation in comparison with the UUO group (29938.02 vs $31854.38 \mu \mathrm{m}^{2}, P<0.05$ ). In contrast, GA and GV were enhanced in a time-dependent manner in the UUO treated with CNP group in which the difference reached significance at 3 weeks and 1 month after ligation when compared with the UUO group (4276.80 vs $3249.51 \mu \mathrm{m}^{2}$ at 3 weeks after ligation for GA; $4035.04 v s$ $2480.62 \mu \mathrm{m}^{2}$ at 1 month after ligation for GA; 133609.23 vs $116571.82 \mu \mathrm{m}^{3}$ at 3 weeks after ligation for GV; 139852.24 vs $111354.75 \mu \mathrm{m}^{3}$ at 1 month after ligation for GV; $P<0.05$; Table 3).

Real-time PCR analysis of CNP, MMP-2, MMP-9, TIMP-1, TIMP-2, and Col-IV mRNA are shown in Figure 3. The relative quantitation of CNP, MMP-2, and MMP-9 transcript were significantly higher in the obstructed kidneys compared with every corresponding time point in the sham-operation group $(P<0.05)$; these elevations in the UUO group could not be corrected by exogenous CNP infusion. No significant difference in TIMP-1 mRNA expression was observed between the sham-operation group and the UUO group from $72 \mathrm{~h}$ to 1 month after ligation $(P>0.05)$. Distinct from TIMP-1 mRNA expression in the obstructed kidneys, the relative quantitation of TIMP-2 transcript augmented in a time-dependent manner and reached significance at 3 weeks after ligation $(P<0.05)$. Obvious elevated renal expression of Col-IV mRNA was observed in the UUO rats as early as $72 \mathrm{~h}$ after ligation and was persistent for 1 month after ligation $(P<0.05)$. CNP administration could significantly reduce the relative quantitation of TIMP-1, TIMP-2, and Col-IV transcript in the UUO treated with CNP group at 3 weeks and 
Table 3 Stereological evaluation among the three groups

Tubulointerstitial area $\left(\mu \mathrm{m}^{2}\right)$

$\begin{array}{ll}\text { Sham-operation group } & \\ 72 \mathrm{~h} & 29402.51 \pm 814.37 \\ 1 \text { Week } & 29361.18 \pm 637.11 \\ 2 \text { Weeks } & 29423.31 \pm 693.81 \\ 3 \text { Weeks } & 29492.10 \pm 808.34 \\ 1 \text { Month } & 29642.35 \pm 761.50\end{array}$

UUO group

$72 \mathrm{~h}$

$29920.33 \pm 900.05$

1 Week

2 Weeks

3 Weeks

1 Month

UUO treated with CNP group

$72 \mathrm{~h}$

$29600.23 \pm 809.26$

1 Week

$29584.35 \pm 764.11$

2 Weeks

$30070.87 \pm 695.79$

3 Weeks

1 Month
$30354.74 \pm 774.17$

$30524.23 \pm 756.95$

$30970.99 \pm 768.14$

$31854.38 \pm 699.49^{a}$
Glomerular area $\left(\mu \mathrm{m}^{2}\right)$

$4318.16 \pm 539.57$

$4701.40 \pm 418.84$

$4413.17 \pm 402.51$

$4498.54 \pm 497.09$

$4312.38 \pm 423.31$

$4462.15 \pm 502.18$

$3978.76 \pm 474.17$

$3809.27 \pm 438.52$

$3249.51 \pm 305.45^{\mathrm{a}}$

$2480.62 \pm 493.05^{\mathrm{a}}$
Glomerular volume $\left(\mu \mathrm{m}^{3}\right)$

$147052.23 \pm 2655.77$

$150352.98 \pm 2040.45$

$148042.34 \pm 3289.65$

$148332.75 \pm 3116.58$

$150111.92 \pm 2038.94$

${ }^{2} P<0.05$, significantly different from the corresponding sham-operation group.

${ }^{\mathrm{b}} P<0.05$, significantly different from the corresponding UUO group.

1 month after ligation compared with the UUO group (for TIMP-1 mRNA expression: a 21\% decrease at 3 weeks after ligation and a $31 \%$ decrease at 1 month after ligation; for TIMP-2 mRNA expression: a $22 \%$ decrease at 3 weeks after ligation and a $24 \%$ decrease at 1 month after ligation; for Col-IV mRNA expression: a 22\% decrease at 3 weeks after ligation and a $27 \%$ decrease at 1 month after ligation; $P<0.05)$. These above expression characterizations of CNP, MMP-2, MMP-9, TIMP-1, TIMP-2, and Col-IV mRNA were also confirmed using western blotting (Figure 4).

\section{DISCUSSION}

Tubulointerstitial fibrosis (TIF) is a life-threatening disease characterized by excess accumulation of ECM and irreversible loss of renal function. ${ }^{28}$ The pathophysiological mechanisms involved in the initiation of TIF are complex. Endothelial dysfunction is one of the common pathways leading to renal failure via TIF, decreasing the production of vasodilators as well as increasing vasoconstrictors. These areas may become devoid of peritubular capillary blood supply and oxygenation to the corresponding region that eventually acts as a profibrogenic stimulus. ${ }^{29}$ Thus, a therapeutic strategy that can redress the imbalance between endothelial vasodilators and vasoconstrictors may be effective for the treatment of TIF. CNP is an endothelium-derived relaxing factor that induces local vasodilation and prevents cell proliferation in a paracrine manner. ${ }^{30}$ Our previous study demonstrated that renal expressions of CNP mRNA and protein tended to be higher in the acute stage of TIF, but progressively declined in the advanced stage. ${ }^{11}$ In this context, we hypothesize that CNP supplementation may be a promising strategy for the treatment of TIF.

UUO is a relevant model in which to provide a valid analog for human TIF. ${ }^{31}$ In this study, we infused the exgenous CNP into the UUO rats. Taking into account its short circulatory half-life and rapid degradation by NEP, ${ }^{32}$ a continuous infusion of CNP $(0.05 \mu \mathrm{g} / \mathrm{kg} / \mathrm{min} \times 1 \mathrm{~h})$ was given once a day from the day of the disease induction until the day of killing. Our study showed that the plasma CNP levels significantly increased in the UUO rats during CNP infusion, up to a maximum of $\sim 10$ times the baseline values. These results are consistent with several previous studies on humans. La Villa et a ${ }^{33,34}$ assayed the levels of circulating CNP in six healthy volunteers who received the continuous infusion of $\mathrm{CNP}(0,2$, and $4 \mathrm{pmol} / \mathrm{min} / \mathrm{kg}$ for $1 \mathrm{~h}$ each) and noted a 40-fold higher plasma CNP levels compared with the baseline values. Therefore, continuous infusion of CNP 
can successfully create a condition with high circulating concentrations.

The first purpose of this study was to determine whether CNP is capable of preventing the development of TIF in vivo. We found that CNP administration could significantly decrease the BUN and Cr levels in the UUO rats from 2 weeks to

a

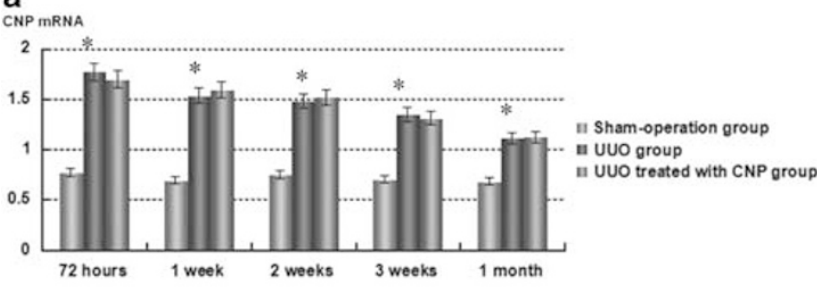

b

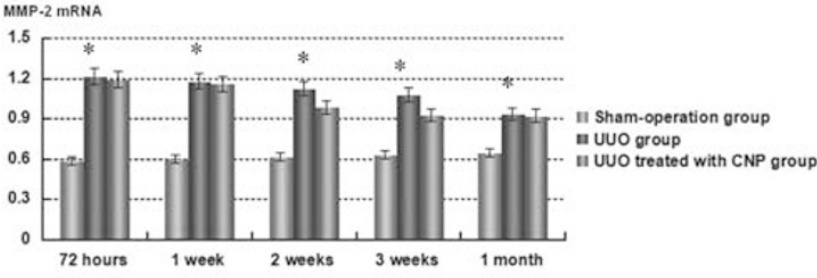

c

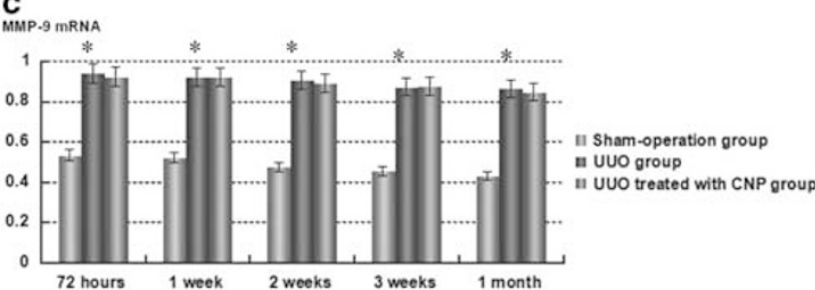

d

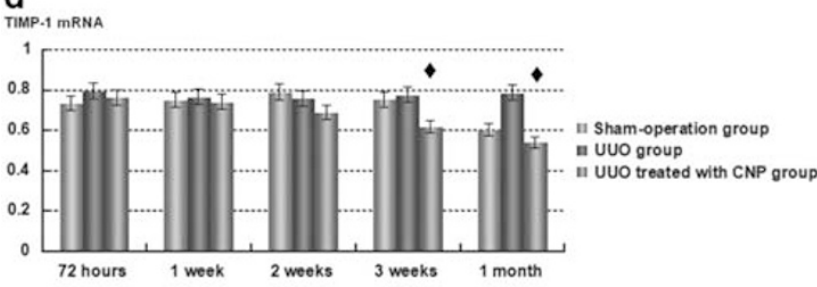

e

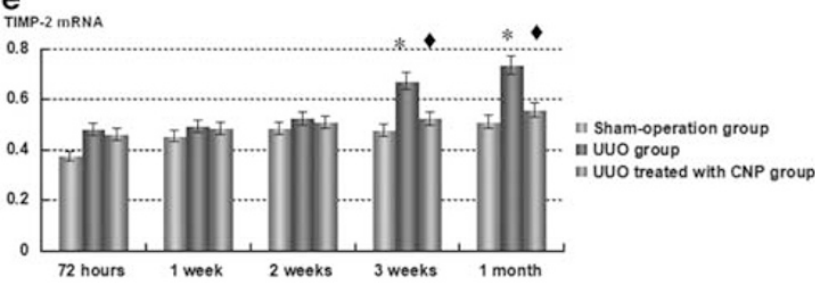

f

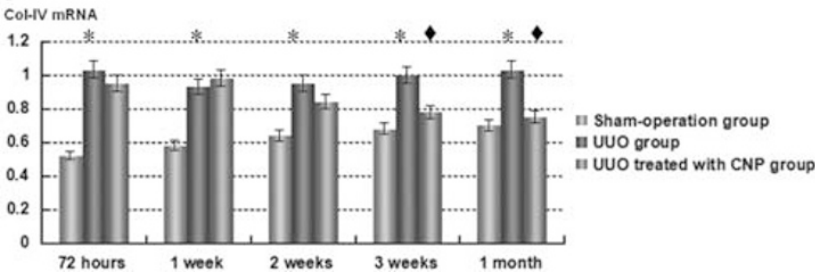

1 month after ligation. Similarly, Pham et al ${ }^{35}$ revealed that infusion of CNP in normal humans was associated with increases in glomerular filtration rate and filtration fraction. The renal protective effects of CNP may be attributed to the local vasorelaxation and hemodynamic improvement through at least four distinct pathways. First, CNP interacts with NPR-C receptor coupled via $G$ proteins, leading to the activation of $\mathrm{Ca}^{2+}$-calmodulin-dependent endothelial nitric oxide synthase and increasing nitric oxide generation that would induce the relaxation of vascular smooth muscle. ${ }^{36}$ Second, $\mathrm{CNP}$ has also been shown to enhance potassium channel activity in vitro, resulting in hyperpolarization of vascular smooth muscle cells. In contrast, the CNP-induced vasodilation can be inhibited with large-conductance calcium-activated potassium channel inhibitors and ATP-dependent potassium channel inhibitors. ${ }^{37}$ Third, CNP infusion can increase local plasma concentrations of ANP through competitive displacement of ANP from natriuretic peptide clearance receptors on the vascular smooth muscle cells. Therefore, in theory, vasodilator effects of CNP in part may be due to increased local ANP plasma levels. ${ }^{38}$ Finally, CNP suppresses the vasoconstrictive effect of angiotensin I but not that of angiotensin II, suggesting that CNP may act as an endogenous inhibitor of vascular angiotensin-converting enzyme. ${ }^{39}$ Interestingly, our results also demonstrated that the protection of CNP was seen predominantly in the late phases, starting from 2 weeks and beyond. BUN and $\mathrm{Cr}$ were adopted to evaluate renal function in the present study, but their sensibilities were limited to reflect the tiny change of renal function. Therefore, more sensitive indexes, such as glomerular filtration rate and cystatin C, should be used in future study. In addition, the protective effects of CNP may need time accumulation.

The common pathological features observed in TIF are infiltration of inflammatory cells, including activated macrophages and fibroblast proliferation with increased amounts of ECM. ${ }^{40}$ In addition, our previous study found that the glomerular size gradually enhanced in a time-dependent manner in the contralateral kidneys, which is capable of

Figure 3 Real-time PCR analysis of CNP, MMP-9, TIMP-1, TIMP-2 and Col-IV mRNA. (a-c) The relative quantitation levels of CNP, MMP-2 and MMP-9 transcript were significantly higher throughout the obstructed kidneys compared to the corresponding sham-operation group; these elevations in the UUO group could not be corrected by exogenous CNP infusion. (d) No significant difference in TIMP-1 mRNA expression was observed between the sham-operation group and the UUO group from $72 \mathrm{~h}$ to 1 month post ligation. (e) The relative quantitation of TIMP-2 transcript augmented in a time-dependent manner and initially reached significance from 3 weeks post ligation. (f) An outstanding elevated renal expression of Col-IV mRNA was observed in the UUO rats as early as $72 \mathrm{~h}$ post ligation and persistent to 1 month post ligation. (d-f) CNP administration could significantly reduce the relative quantitation of TIMP-1, TIMP-2 and Col-IV transcript in the UUO treated with the CNP group at 3 weeks and 1 month post ligation compared to the UUO group. ${ }^{*} P<0.05$, significantly different from the corresponding shamoperation group; $\bullet<0.05$, significantly different from the corresponding UUO group. 


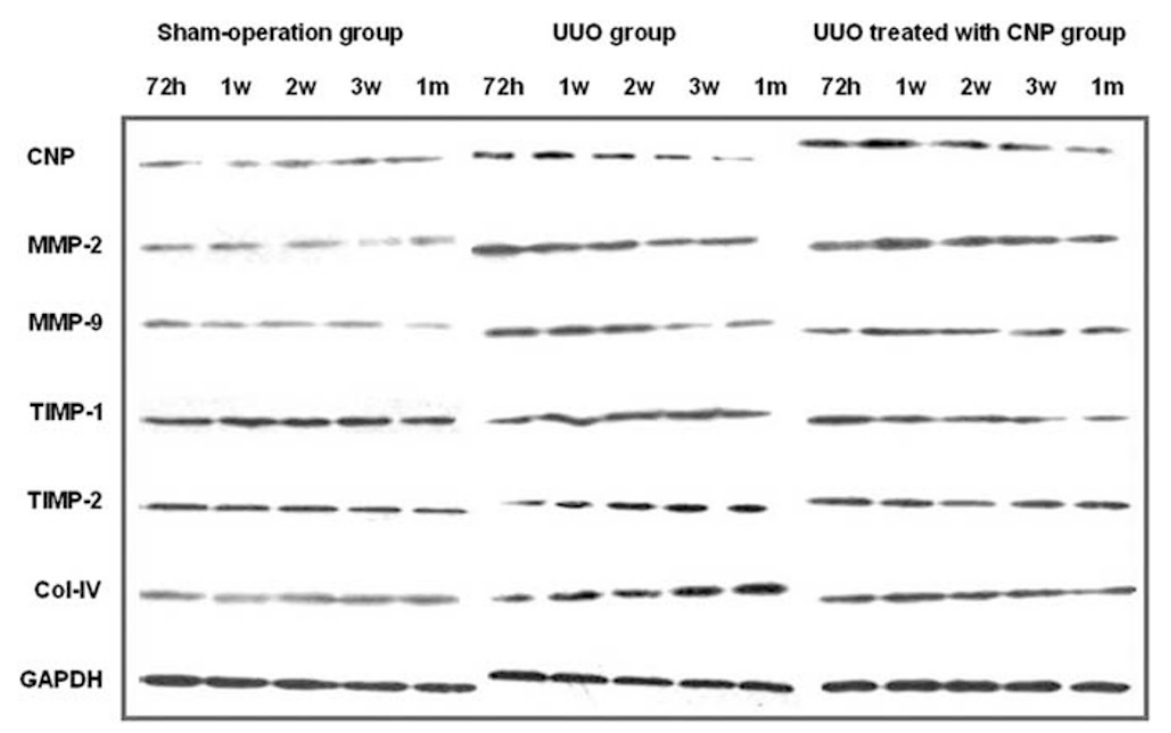

Figure 4 Western blot identification of CNP, MMP-2, MMP-9, TIMP-1, TIMP-2, Col-IV, and GAPDH protein.

compensating for the damaged renal unit of the obstructed kidneys. ${ }^{20}$ However, in this study, UUO rats exhibited significant higher BUN and $\mathrm{Cr}$ than those in the shamoperation group from 2 weeks to 1 month after ligation; therefore, we believe that systemic inflammation and oxidative stress generated by UUO may mainly contribute to renal damage. ${ }^{41,42}$ In this study, in addition to renal protective effects, CNP infusion could also alleviate these above pathological lesions in the obstructed kidneys. More specifically, stereological evaluation disclosed that CNP infusion caused significantly low TA, whereas high GA and GV in the obstructed kidneys. The excessive Col-IV mRNA and protein in the obstructed kidneys could be prevented by CNP infusion in the advanced stages, according to real-time PCR and western blot analysis, respectively. We confirmed previous findings that CNP may represent a downregulator of renal ECM synthesis both in vivo and in vitro. CanaanKuhl et $a l^{43}$ investigated the effects of exogenous CNP administration on mesangial cell proliferation and ECM accumulation in the rat model of anti-Thy 1.1 mesangioproliferative nephritis in vivo, and found that the renal immunostaining score for Col-IV and fibronectin were significantly reduced in the CNP group as compared with buffer-treated rats on day 8 after disease. Furthermore, in the report of Osawa et al, ${ }^{44} \mathrm{CNP}$ could also inhibit Col-IV expression in cultured human mesangial cells. Beyond our expectation, in this study, we also found that there was prominent tubular dilatation in the UUO rats treated with CNP. However, the underlying mechanisms are unclear.

Thereafter, the second purpose of this study was to probe whether the downregulation of renal ECM synthesis in CNPtreated animals was mediated by MMP/TIMP pathway. MMP-2 and MMP-9 are involved in the degradation of ColIV, a major component of renal tubulointerstitium. ${ }^{45}$ TIMPs can specifically combine with the catalytic center of zinc ion and close their catalytic activities. Among them, TIMP-1 binds to MMP-9 and TIMP-2 can complex with MMP-2. ${ }^{46}$ Our previous studies in humans and experimental animals have demonstrated that MMP-2, MMP-9, TIMP-1, and TIMP-2 participate in the occurrence and progression of diverse nephropathies. ${ }^{4-49}$ In general, the balance of MMP/ TIMP metabolism is shifted toward the reduction in MMP-2 and MMP-9 activity and/or the increment in TIMP-1 and TIMP-2 expression under pathological situations. Therefore, any measure that reverses these shift may be beneficial in retarding the progression of TIF. In this study, we focused on the renal expression of MMP-2, MMP-9, TIMP-1, and TIMP2 in the UUO rats treated with exogenous CNP, and found that CNP could significantly reduce TIMP-1 and TIMP-2 expression at 3 weeks and 1 month after ligation, but did not play any role in MMP-2 and MMP-9 expression.

In conclusion, this study shows that exogenous CNP infusion can ameliorate UUO-induced TIF in rats. The renal protective effects of CNP may be attributed to the local vasorelaxation and hemodynamic improvement. In addition, CNP infusion can also alleviate the pathological lesions and ECM accumulation in the obstructed kidneys through downregulation of TIMP-1 and TIMP-2 expression. Nevertheless, the use of CNP as a therapeutic agent requires further evaluation before being considered for human TIF.

\section{ACKNOWLEDGMENTS}

This study was supported by the National Natural Science Foundation of China (No. 81000306) and the Post-Doctoral Foundation of Anhui Medical University (No. 2009KJ02).

\section{DISCLOSURE/CONFLICT OF INTEREST}

The authors declare no conflict of interest. 
1. Stryjewski PJ, Nessler B, Cubera K, et al. Natriuretic peptides. History of discovery, chemical structure, mechanism of action and the removal routes. Basis of diagnostic and therapeutic use. Przegl Lek 2013;70:463-467.

2. Sudoh T, Minamino N, Kangawa K, et al. C-type natriuretic peptide (CNP): a new member of the natriuretic peptide family identified in porcine brain. Biochem Biophys Res Comm 1990;168:863-870.

3. Leuranguer V, Vanhoutte PM, Verbeuren T, et al. C-type natriuretic peptide and endothelium-dependent hyperpolarization in the guineapig carotid artery. Br J Pharmacol 2008;153:57-65.

4. Del Ry S, Cabiati M, Vozzi F, et al. Expression of C-type natriuretic peptide and its receptor NPR-B in cardiomyocytes. Peptides 2011;32 1713-1718.

5. Suda M, Tanaka K, Fukushima M, et al. C-type natriuretic peptide as an autocrine/paracrine regulator of osteoblast. Evidence for possible presence of bone natriuretic peptide system. Biochem Biophys Res Commun 1996;223:1-6.

6. Totsune K, Takahashi K, Murakami O, et al. Immunoreactive C-type natriuretic peptide in human adrenal glands and adrenal tumors. Peptides 1994;15:287-290.

7. Nielsen SJ, Gøtze JP, Jensen HL, et al. ProCNP and CNP are expressed primarily in male genital organs. Regul Pept 2008;146:204-212.

8. Kalra PR, Clague JR, Coats AJ, et al. C-type natriuretic peptide production by the human kidney is blunted in chronic heart failure. Clin Sci (Lond) 2009;118:71-77.

9. Prickett TC1 Charles CJ, Yandle TG, et al. Skeletal contributions to plasma CNP forms: evidence from regional sampling in growing lambs. Peptides 2009;30:2343-2347.

10. Hu P, Wang J, Zhao XQ, et al. Overexpressed C-type natriuretic peptide serves as an early compensatory response to counteract extracellular matrix remodeling in unilateral ureteral obstruction rats. Mol Biol Rep 2013;40:1429-1441.

11. Hu P, Zhao XQ, Wang J, et al. Paradoxical expressions of natriuretic peptide receptor- $C$ and neutral endopeptidase account for C-type natriuretic peptide decline during the progression of experimental obstructive nephropathy. J Renin Angiotensin Aldosterone Syst; advance online publication, 4 November 2013 (e-pub ahead of print)

12. Hu P, Wang J, Hu B, et al. Increased urinary C-type natriuretic peptide excretion may be an early marker of renal tubulointerstitial fibrosis. Peptides 2012;37:98-105.

13. Villar IC, Panayiotou CM, Sheraz A, et al. Definitive role for natriuretic peptide receptor-C in mediating the vasorelaxant activity of C-type natriuretic peptide and endothelium-derived hyperpolarising factor. Cardiovasc Res 2007:74:515-525.

14. Komatsu $\mathrm{Y}$, Itoh $\mathrm{H}$, Suga $\mathrm{S}$, et al. Regulation of endothelial production of C-type natriuretic peptide in coculture with vascular smooth muscle cells. Role of the vascular natriuretic peptide system in vascular growth inhibition. Circ Res 1996;78:606-614.

15. Doi $\mathrm{K}$, Ikeda $\mathrm{T}$, Itoh $\mathrm{H}$, et al. C-type natriuretic peptide induces redifferentiation of vascular smooth muscle cells with accelerated reendothelialization. Arterioscler Thromb Vasc Biol 2001;21:930-936.

16. Hayashi MA, Murbach AF, lanzer $\mathrm{D}$, et al. The C-type natriuretic peptide precursor of snake brain contains highly specific inhibitors of the angiotensin-converting enzyme. J Neurochem 2003;85:969-977.

17. Izumiya $\mathrm{Y}$, Araki S, Usuku H, et al. Chronic C-type natriuretic peptide infusion attenuates angiotensin II-induced myocardial superoxide production and cardiac remodeling. Int J Vasc Med 2012;2012:246058.

18. Itoh $\mathrm{T}$, Nagaya $\mathrm{N}$, Murakami $\mathrm{S}$, et al. C-type natriuretic peptide ameliorates monocrotaline-induced pulmonary hypertension in rats. Am J Respir Crit Care Med 2004;170:1204-1211.

19. Del Ry $S$, Maltinti $M$, Emdin $M$, et al. Radioimmunoassay for plasma C-type natriuretic peptide determination: a methodological evaluation. Clin Chem Lab Med 2005;43:641-645.

20. Hu $\mathrm{P}, \mathrm{Zhao} \mathrm{XQ}$, Wang J, et al. Simplified stereological evaluation of renal morphology after unilateral ureteral obstruction. Int J Med Sci 2013;10:385-391.

21. Bae EH, Kim SW. Upregulation of renin-angioteinsin, endothelin and C-type natriuretic peptide in rat glomerulus with bilateral ureteral obstruction. Korean J Physiol Pharmacol 2006;10:343-348.

22. Garvey SM, Sinden DS, Schoppee Bortz PD, et al. Cyclosporine upregulates Krüppel-like factor-4 (KLF4) in vascular smooth muscle cells and drives phenotypic modulation in vivo. J Pharmacol Exp Ther 2010;333:34-42.
23. Oin $\mathrm{YH}$, Lei $\mathrm{FY}$, Hu $\mathrm{P}$, et al. Effect of all-trans retinoic acid on renal expressions of matrix metalloproteinase-2, matrix metalloproteinase- 9 and tissue inhibitor of metalloproteinase- 1 in rats with glomerulosclerosis. Pediatr Nephrol 2009;24:1477-1486.

24. Schäfer S, Ellinghaus $\mathrm{P}$, Janssen $\mathrm{W}$, et al. Chronic inhibition of phosphodiesterase 5 does not prevent pressure-overload-induced right-ventricular remodelling. Cardiovasc Res 2009;82:30-39.

25. Bowman CJ, Turner KJ, Sar M, et al. Altered gene expression during rat Wolffian duct development following di(n-butyl) phthalate exposure. Toxicol Sci 2005;86:161-174.

26. Nagai $\mathrm{Y}$, Yao L, Kobori $\mathrm{H}$, et al. Temporary angiotensin II blockade at the prediabetic stage attenuates the development of renal injury in type 2 diabetic rats. J Am Soc Nephrol 2005;16:703-711.

27. Schmittgen TD, Livak KJ. Analyzing real-time PCR data by the comparative $C(T)$ method. Nat Protoc 2008;3:1101-1108.

28. Farris $A B$, Colvin RB. Renal interstitial fibrosis: mechanisms and evaluation. Curr Opin Nephrol Hypertens 2012;21:289-300.

29. Satoh M. Endothelial dysfunction as an underlying pathophysiological condition of chronic kidney disease. Clin Exp Nephrol 2012;16:518-521.

30. Liang $\mathrm{CF}, \mathrm{Au} \mathrm{AL}$, Leung $\mathrm{SW}$, et al. Endothelium-derived nitric oxide inhibits the relaxation of the porcine coronary artery to natriuretic peptides by desensitizing big conductance calcium-activated potassium channels of vascular smooth muscle. J Pharmacol Exp Ther 2010;334:223-231.

31. Zhou TB, Qin YH, Lei FY, et al. Association of prohibitin-1 and 2 with oxidative stress in rats with renal interstitial fibrosis. Mol Biol Rep 2014:41:3033-3043.

32. Hu P, Lu L, Hu B, et al. Renal action of C-type natriuretic peptide: advocating the isolated perfused rat kidney model. Saudi J Kidney Dis Transpl 2010;21:613-620.

33. La Villa G, Mannelli M, Lazzeri $C$ et al. Different effects of atrial and C-type natriuretic peptide on the urinary excretion of endothelin-1 in man. Clin Sci (Lond) 1998:95:595-602.

34. Barletta G, Lazzeri C, Vecchiarino S, et al. Low-dose C-type natriuretic peptide does not affect cardiac and renal function in humans. Hypertension 1998;31:802-808

35. Pham I, Sediame S, Maistre G, et al. Renal and vascular effects of C-type and atrial natriuretic peptides in humans. Am J Physio 1997;273:R1457-R1464.

36. Brunner F, Wölkart G. Relaxant effect of C-type natriuretic peptide involves endothelium and nitric oxide-cGMP system in rat coronary microvasculature. Cardiovasc Res 2001:51:577-584.

37. Chauhan SD, Nilsson H, Ahluwalia A, et al. Release of C-type natriuretic peptide accounts for the biological activity of endothelium-derived hyperpolarizing factor. Proc Natl Acad Sci USA 2003;100:1426-1431.

38. Honing ML, Smits $\mathrm{P}$, Morrison PJ, et al. C-type natriuretic peptideinduced vasodilation is dependent on hyperpolarization in human forearm resistance vessels. Hypertension 2001:37:1179-1183.

39. Davidson NC, Barr CS, Struthers AD. C-type natriuretic peptide. An endogenous inhibitor of vascular angiotensin-converting enzyme activity. Circulation 1996;93:1155-1159.

40. Zhou TB, Qin YH, Lei FY, et al. Prohibitin attenuates oxidative stress and extracellular matrix accumulation in renal interstitial fibrosis disease. PLoS One 2013;8:e77187.

41. Gonzalez J, Mouttalib S, Delage $C$, et al. Dual effect of chemokine CCL7/MCP-3 in the development of renal tubulointerstitial fibrosis. Biochem Biophys Res Commun 2013;438:257-263.

42. Kawada N, Moriyama T, Ando A, et al. Increased oxidative stress in mouse kidneys with unilateral ureteral obstruction. Kidney Int 1999;56:1004-1013.

43. Canaan-Kühl S, Ostendorf T, Zander K, et al. C-type natriuretic peptide inhibits mesangial cell proliferation and matrix accumulation in vivo. Kidney Int 1998;53:1143-1151.

44. Osawa $\mathrm{H}$, Yamabe $\mathrm{H}$, Kaizuka $\mathrm{M}$, et al. C-Type natriuretic peptide inhibits proliferation and monocyte chemoattractant protein-1 secretion in cultured human mesangial cells. Nephron 2000;86:467-472.

45. Lelongt B, Trugnan G, Murphy G, et al. Matrix metalloproteinases MMP2 and MMP9 are produced in early stages of kidney morphogenesis but only MMP9 is required for renal organogenesis in vitro. J Cell Biol 1997:136:1363-1373.

46. Sekiuchi M, Kudo A, Nakabayashi $\mathrm{K}$, et al. Expression of matrix metalloproteinases 2 and 9 and tissue inhibitors of matrix metalloproteinases 2 and 1 in the glomeruli of human glomerular 
diseases: the results of studies using immunofluorescence, in situ hybridization, and immunoelectron microscopy. Clin Exp Nephrol 2012;16:863-874.

47. Hu P, Qin YH, Pei J, et al. Beneficial effect of all-trans retinoic acid (ATRA) on glomerulosclerosis rats via the down-regulation of the expression of alpha-smooth muscle actin: a comparative study between ATRA and benazepril. Exp Mol Pathol 2010;89:51-57.
48. Hu P, Xuan $\mathrm{Q}, \mathrm{Hu}$ B, et al. Anti-neutral endopeptidase, natriuretic peptides disarrangement, and proteinuria onset in membranous nephropathy. Mol Biol Rep 2013;40:2963-2967.

49. Qin YH, Zhou TB, Lei FY, et al. Cut-off values for serum matrix metalloproteinase-9: is there a threshold to predict renal involvement for Henoch-Schonlein purpura in children? Nephrology (Carlton) 2011;16:93-99. 